\title{
Swimming exercise reverses aging-related contractile abnormalities of female heart by improving structural alterations
}

\author{
Nihal Ozturk ${ }^{1}$, Yusuf Olgar ${ }^{1}$, Hakan $\mathrm{Er}^{1,2}$, Murathan Kucuk ${ }^{3}$, Semir Ozdemir ${ }^{1}$ \\ ${ }^{1}$ Department of Biophysics, Akdeniz University Faculty of Medicine, Antalya, Turkey \\ ${ }^{2}$ Electron Microscopy Unit, Akdeniz University Faculty of Medicine, Antalya, Turkey \\ ${ }^{3}$ Department of Cardiology, Akdeniz University Faculty of Medicine, Antalya, Turkey
}

\begin{abstract}
Background: The objective of this study was to examine the effect of swimming exercise on aging-related $\mathrm{Ca}^{2+}$ handling alterations and structural abnormalities of female rat heart.

Methods: For this purpose, 4-month and 24-month old female rats were used and divided into three following groups: sedentary young (SY), sedentary old (SO), and exercised old (Ex-O). Swimming exercise was performed for 8 weeks (60 min/day, 5 days/week). Myocyte shortening, L-type Ca ${ }^{2+}$ currents and associated $\mathrm{Ca}^{2+}$ transients were measured from ventricular myocytes at $36 \pm 1^{\circ} \mathrm{C} . \mathrm{NOX}-4$ levels, aconitase activity, glutathione measurements and ultrastructural examination by electron microscopy were conducted in heart tissue.

Results: Swimming exercise reversed the reduced shortening and slowed kinetics of aged cardiomyocytes. Although the current density was similar for all groups, $\mathrm{Ca}^{2+}$ transients were higher in $\mathrm{SO}$ and Ex-O myocytes with respect to the SY group. Caffeine-induced $\mathrm{Ca}^{2+}$ transients and the integrated NCX current were lower in cardiomyocytes of SY rats compared with other groups, suggesting an increased sarcoplasmic reticulum $\mathrm{Ca}^{2+}$ content in an aged heart. Aging led to upregulated cardiac NOX-4 along with declined aconitase activity. Although it did not reverse these oxidative parameters, swimming exercise achieved a significant increase in glutathione levels and improved structural alterations of old rats' hearts.
\end{abstract}

Conclusions: We conclude that swimming exercise upregulates antioxidant defense capacity and improves structural abnormalities of senescent female rat heart, although it does not change $\mathrm{Ca}^{2+}$ handling alterations further. Thereby, it improves contractile function of aged myocardium by mitigating detrimental effects of oxidative stress. (Cardiol J 2017; 24, 1: 85-93)

Key words: aging, swimming exercise, myocardial structure, oxidative stress, female heart

\section{Introduction}

Numerous studies in human and animal subjects have shown that aging may cause structural and functional alterations in the myocardium. These age-dependent changes lead to a prominent decline in cardiac function, which may cause elderly people to be more prone to various cardiovascular diseases. The cardiac contraction- relaxation cycle has been shown to alter with age, most likely due to dysregulation of cytosolic calcium homeostasis [1, 2]. In cardiomyocytes, a typical contraction-relaxation cycle is initiated by opening of L-type $\mathrm{Ca}^{2+}$ channel (LTCC) which triggers the opening of the ryanodine receptor (RYR) and gives rise to a global intracellular $\mathrm{Ca}^{2+}$ increase. This is then terminated by removal of excess of cytosolic calcium via either the sarco-

Address for correspondence: Dr. Semir Ozdemir, Akdeniz University Faculty of Medicine, Department of Biophysics, Antalya, Turkey, tel: +90 242 2496907, e-mail: e-mail: osemir@akdeniz.edu.tr

Received: 11.04.2016 Accepted: 29.07.2016 
plasmic reticulum (SR) $\mathrm{Ca}^{2+}$ pump (SERCA2a) to SR or by the $\mathrm{Na}^{+} / \mathrm{Ca}^{2+}$ exchanger (NCX) to the extracellular space. In aging hearts, however, decreased $\mathrm{Ca}^{2+}$ removal rate and altered $\mathrm{Ca}^{2+}$ handling which precede slowed contraction may eventually result in ventricular dysfunction [1]. Despite this hypothesis, somewhat unchanged [3], depressed $[4,5]$ or increased [6] results have been reported for cell shortening and $\mathrm{Ca}^{2+}$ transient amplitude in animal models of senescent heart. The most likely mechanisms that account for these alterations in cardiac excitation-contraction (E-C) coupling are L-type $\mathrm{Ca}^{2+}$ current $\left(\mathrm{I}_{\mathrm{CaL}}\right)$ and altered activity or expression of $\mathrm{Ca}^{2+}$ regulatory proteins which may lead to impaired SR function. However, previous studies performed in different species have reported [7, 8] unchanged [9] or increased [10] $\mathrm{I}_{\mathrm{CaL}}$ densities with age in cardiac myocytes.

Although the exact mechanism by which aging impairs intracellular $\mathrm{Ca}^{2+}$ homeostasis and cardiac contractile function has not been fully elucidated, the accumulation of reactive oxygen species (ROS) has been raised as the contributing process [11]. In fact, apart from the leakage from mitochondrial electron transport chain (ETC), ROS can be generated through cellular enzymes including NOX-4, upregulation of which is suggested to inactivate aconitase and thereby play an important role in mitochondrial aging $[12,13]$.

On the other hand, the effect of chronic exercise on electrical and mechanical activities of heart is still an area of interest. It has been demonstrated that chronic exercise is capable of reversing the aging-related prolongation of relaxation time and $\mathrm{Ca}^{2+}$ transient in papillary muscle due to improvement of $\mathrm{Ca}^{2+}$ reuptake by cardiac SR $[14,15]$. However, the influence of swimming exercise on aging-related changes in $\mathrm{Ca}^{2+}$ transients and underlying regulatory mechanisms is still uncertain. Secondly, current findings that address either age-related or exercise-induced changes in myocardial function have mostly been obtained from male subjects, despite the fact that recent studies have shown striking behavioral differences between male and female myocardium $[7,16]$. Therefore, the purpose of this study was to determine whether swimming exercise elicits any alteration in ultrastructure and $\mathrm{Ca}^{2+}$ handling of senescent female rat myocardium and to delineate the underlying cellular mechanism. In order to examine the potential role of oxidative stress in aging-related changes of female myocardium NOX-4, GSH/GSSG levels and aconitase activity were also studied.

\section{Methods}

\section{Animals}

In this study, 4- and 24-month-old female Wistar rats were used. They were housed at $23 \pm$ $\pm 2^{\circ} \mathrm{C}$ and had free access to standard rat chow and drinking water. Rats were assigned to three groups as: sedentary young (SY, $\mathrm{n}=19$ ), sedentary old $(\mathrm{SO}, \mathrm{n}=21)$, and exercised old $(\mathrm{Ex}-\mathrm{O}, \mathrm{n}=20)$. The animals in training group were subjected to swimming exercise $(60 \mathrm{~min} /$ day, 5 days/week for 8 weeks $)$ in a glass chamber $(100 \times 50 \mathrm{~cm}$ diameter and $50 \mathrm{~cm}$ depth) filled with tap water and maintained at $33 \pm 2^{\circ} \mathrm{C}$. For adaptation, duration of the first swimming experience was limited to $10 \mathrm{~min}$ and increased by $10 \mathrm{~min}$ daily until $60 \mathrm{~min}$ was reached. The experimental protocol was approved by the Animal Care and Usage Committee of Akdeniz University and in accordance with the Declaration of Helsinki and International Association for the Study of Pain (IASP) guidelines.

\section{Cell isolation}

The rats were anaesthetized with pentobarbital sodium $(50 \mathrm{mg} / \mathrm{kg}$ body weight, intraperitoneal) and their hearts were excised rapidly. The cell isolation was carried out as previously described [17, 18]. Briefly, the aorta was cannulated and perfused retrogradely through the coronary artery with a $\mathrm{Ca}^{2+}$-free solution containing (in mM): $137 \mathrm{NaCl}, 5.4 \mathrm{KCl}, 1.2 \mathrm{MgSO}_{4}$, $1.2 \mathrm{KH}_{2} \mathrm{PO}_{4}, 6$ HEPES, 20 glucose (pH 7.2) and bubbled with $100 \% \mathrm{O}_{2}$ at $37^{\circ} \mathrm{C}$. This was followed by a perfusion of hearts with the same solution containing $0.7-0.8 \mathrm{mg} / \mathrm{mL}$ collagenase and $0.07 \mathrm{mg} / \mathrm{mL}$ protease. Left ventricles were then removed, minced into small pieces and gently massaged through a nylon mesh. Subsequently, the cell suspension was washed several times and $\mathrm{Ca}^{2+}$ was increased in a graded manner for adaptation. All experiments were performed at $36 \pm 1^{\circ} \mathrm{C}$.

\section{Myocyte shortening}

Myocyte contraction was measured by detecting the length of two edges with contractility recording system (IonOptix LLC, Milton USA) during electrical field stimulation ( $5 \mathrm{~V}$ and $1 \mathrm{~Hz})$. The Soft-edge software (IonOptix) was used to capture and analyze the changes in edge length $\left(\mathrm{L} / \mathrm{L}_{0}\right)$ and results given as fractional shortening. 


\section{Voltage clamp recordings}

Standard Tyrode solution which contained (in $\mathrm{mmol} / \mathrm{L}$ ) $137 \mathrm{NaCl}, 5.4 \mathrm{KCl}, 0.5 \mathrm{MgCl}_{2}$, 1.5 $\mathrm{CaCl}_{2}, 11.8 \mathrm{HEPES}$, and 10 glucose (pH adjusted to 7.40 with $\mathrm{NaOH}$ ) was used as external solution. For measurement of $\mathrm{I}_{\mathrm{CaL}}$, pipettes were filled with a solution containing (in mmol/L) $120 \mathrm{Cs}$-aspartate, $20 \mathrm{KCl}, 10 \mathrm{NaCl}, 10 \mathrm{HEPES}, 5 \mathrm{MgATP}$, and 0.05 Fura-2 pentapotassium salt $(\mathrm{pH}=7.2$ with $\mathrm{CsOH})$. During the measurement of $\mathrm{I}_{\mathrm{Ca}}, \mathrm{K}^{+}$was replaced with $\mathrm{Cs}^{+}$in the external Tyrode solution.

Currents were recorded by patch-clamp amplifier (Axon 200B, Molecular Devices, USA) using the whole-cell configuration mode. Following a pre-pulse from $-70 \mathrm{mV}$ to $-45 \mathrm{mV}$ for inactivation of $\mathrm{Na}^{+}$currents, $300 \mathrm{~ms}$ steps ranging between $-50 \mathrm{mV}$ and $+60 \mathrm{mV}$ were applied at $0.2 \mathrm{~Hz}$. $\mathrm{I}_{\mathrm{CaL}}$ was calculated as the difference between the peak current and the end of pulse current. Current densities were normalized to the relevant cell capacitance and presented as $\mathrm{pA} / \mathrm{pF}$.

The ratio of the fluorescence emission at $510 \mathrm{~nm}$ in response to $340 \mathrm{~nm}$ and $380 \mathrm{~nm}$ excitation wavelengths was used as an indicator of intracellular $\mathrm{Ca}^{2+}$ (IonOptix, Milton, USA). The Ionwizard software was used to capture and analyze the $\mathrm{Ca}^{2+}$ transients.

The $\mathrm{Ca}^{2+}$ content of the SR were measured during application of $10 \mathrm{mmol} / \mathrm{L}$ caffeine via fast perfusion system (VC-6 Fast Perfusion System, Harvard Apparatus, USA).

\section{Histopathological examination by transmission electron microscopy}

For electron microscopic examination, left ventricular tissues were fixed in $4 \%$ glutaraldehyde, $0.1 \mathrm{M}$ Sorensen phosphate buffer $(\mathrm{pH}=7.4)$ at $4^{\circ} \mathrm{C}$ for $2 \mathrm{~h}$ and then in $1 \%$ phosphate-buffered osmium tetroxide for $2 \mathrm{~h}$. The specimens were dehydrated in a graded series of ethanol and embedded in Araldite CY212 epoxy resin (TAAB, England). Semi-thin $(1 \mathrm{~mm})$ and ultra-thin sections cut by an ultra-microtome (Leica Ultracut UCT, Austria) were stained with uranyl acetate and lead citrate prior to examination by transmission electron microscope (Zeiss LEO 906E TEM, Oberkochen, Germany).

\section{Assessment of NOX-4 content and aconitase activity}

For assessment of the NOX-4 level in heart tissue, an ELISA kit (USCN Life Science Inc., China) was used. Briefly, the tissue homogenate was centrifuged at $5000 \times \mathrm{g}$ and the supernatants were collected and assayed immediately. $100 \mu \mathrm{L}$ samples were added to the wells and incubated for $2 \mathrm{~h}$ at room temperature. Following addition of the reagents reaction was stopped and the absorbance measured by spectrophotometer at wavelength of $450 \mathrm{~nm}$.

Aconitase activity was measured by using the Aconitase Assay Kit (Cayman Chemical Co., USA). Citrate is isomerized by aconitase to isocitrate which is then converted to alpha-ketoglutarate in a reaction catalyzed by isocitric dehydrogenase. These reactions are monitored by measuring the increase in absorbance at $340 \mathrm{~nm}$.

\section{Tissue glutathione and oxidized glutathione levels}

Tissue glutathione (GSH) and oxidized glutathione (GSSG) levels were measured by a commercial assay kit (Cayman Chemical Ann Arbor, MI). Briefly, supernatants were deproteinated in 10\% metaphosphoric acid (Sigma Aldrich, Steinheim, Switzerland) and the GSSG was reduced to GSH in the provided assay cocktail of the kit. The resultant yellow color of this reaction was measured at an absorbance of $405 \mathrm{~nm}$. Tissue levels of GSSG were determined by first derivation of GSH with 2-vinylpyridine (Sigma Aldrich, Steinheim, Switzerland). The values of GSH and GSSG were calculated from their respective slopes for each sample by using a GSSG or GSH standard curve.

\section{Statistical analysis}

Statistical analysis of data was performed by using a one-way ANOVA followed by a post-hoc Bonferroni test for comparison of the relevant groups. Where non-parametric analysis of the groups was more appropriate Kruskal-Wallis test was used. Values smaller than $0.05(\mathrm{p}<0.05)$ were accepted as significant. Data are represented as mean \pm standard error of mean (SEM).

\section{Results}

\section{Morphological parameters}

As it can be seen in Figure 1, body weights of aged groups were significantly higher before the training program and these differences were maintained at the termination of swimming protocol. Swimming exercise did not elicit any significant change in body weights of rats when compared with age-matched controls. The apparent cardiac hypertrophy in the old rats was demonstrated by greater heart weight and heart weight/tibia ratio and this difference did not change with swimming exercise. 


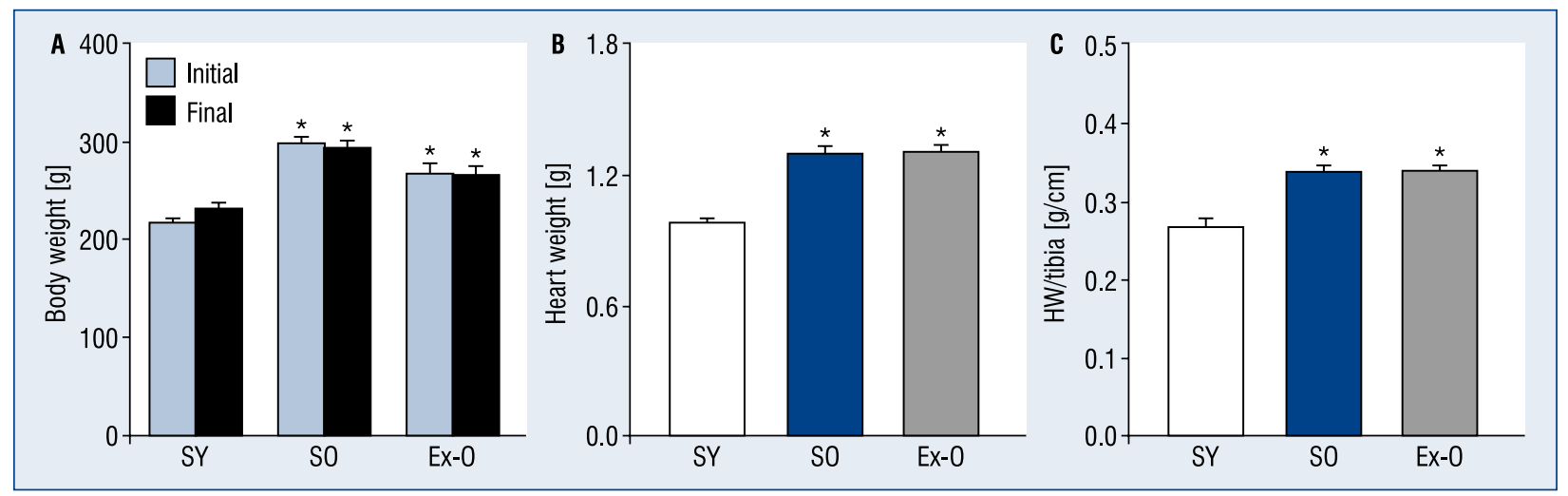

Figure 1. General features of animals studied. Body weight, heart weight (HW), and heart weight/tibia length ratio (HW/ /tibia) of sedentary young (SY), sedentary old (SO), and exercised old (Ex-O) female rats. Heart weight and HW/tibia increase significantly with age and exercise training. Data are presented as mean \pm SEM; ${ }^{*} p<0.05$ vs. SY group ( $n=19,21,20$ rats for SY, SO and Ex-O groups, respectively).

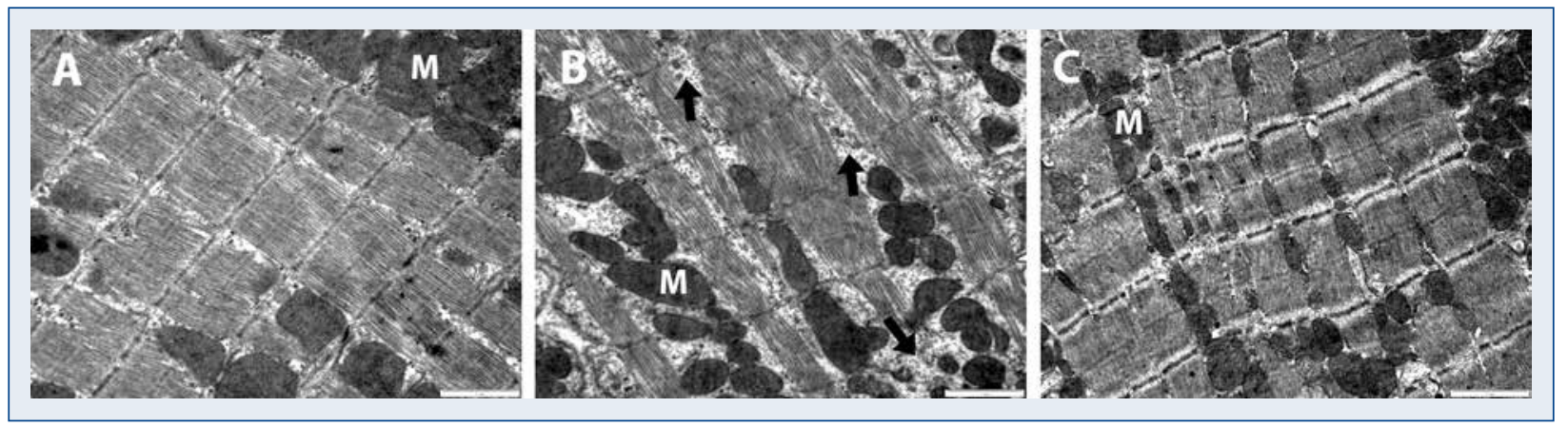

Figure 2. Ultrastructural examinations of hearts by electron microscopy. The micrographs show ultrastructural analysis of myocardium in panels A-C; uranyl acetate/lead citrate stained (scale bar $1 \mu \mathrm{m}$ ) sections from left ventricles of sedentary young (SY), sedentary old (SO), and exercised old (Ex-O) groups. Poorly organized and disrupted myofibrils and thinning of the $Z$ lines were observed in the SO group. Swimming exercise significantly improved these morphological and ultrastructural changes; $\mathrm{M}$ - mitochondria; black arrow - myofibrillar spacing.

\section{Effects of aging and exercise} on cardiac muscle ultrastructure

Ultrastructural examination by electron microscopy demonstrated that $\mathrm{SO}$ group rats show randomly distributed mitochondria between poorly organized and disrupted myofibrils with increased myofibril spacing in an electron-lucent sarcoplasm (Fig. 2B). These abnormalities were significantly reduced in Ex-O rats (Fig. 2C). Sedentary young hearts, however, showed coherent myocardial structure, with myofibrils composed of regular and uninterrupted sarcomeres delimited by $Z$ lines and adjacent myofibrils (Fig. 2A). Swimming exercise induced electron-dense sarcoplasm with prominent rows of mitochondria intervenes between myofibrils in old rats (Fig. 2C).

\section{Contractile functions}

Fractional shortening dramatically diminished in aging group myocytes (SO: $4.56 \pm 0.45, \mathrm{n}=35$ cells from 9 rats; $\mathrm{p}<0.01)$ when compared with the young group (SY: $8.18 \pm 0.53, \mathrm{n}=35$ cells from 9 rats) (Fig. 3A, B). However, exercise training reversed the aging-related contractile deficit (Ex-O: $7.60 \pm 0.51, \mathrm{n}=35$ and $\mathrm{p}<0.01)$. Moreover, rates of contraction $(+\mathrm{dL} / \mathrm{dt})$ and relaxation $(-\mathrm{dL} / \mathrm{dt})$ of the SO group cardiomyocytes were significantly reduced, while swimming exercise restored these impaired kinetics of aged myocytes (Fig. 3C, D). We therefore conclude that exercise training improves contractile parameters of myocardium in aged females. 


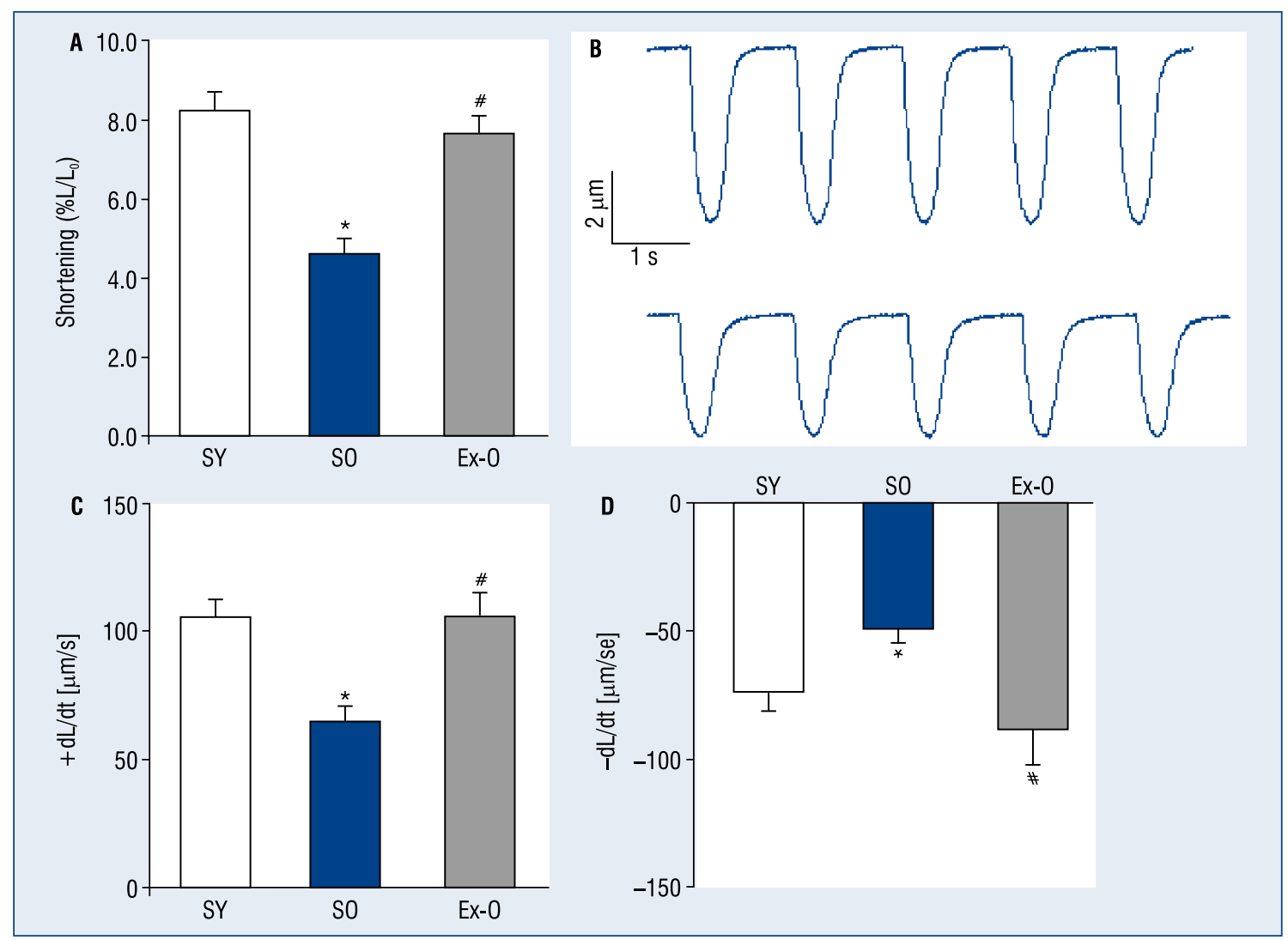

Figure 3. Effect of swimming exercise on aging-related changes in contractile function of myocytes presented as fractional shortening $(\mathbf{A})$, and representative traces (B). Reduced rates of systolic contraction (C), and diastolic relaxation (D) were also improved in Ex-O group ventricular myocytes. Data are presented as mean \pm SEM; ${ }^{*} p<0.01$ vs. SY group; \#p < 0.01 vs. SO group (number of cells, $n=35$ [9 hearts], 35 [7 hearts], 30 [7 hearts] for SY, SO and Ex-O groups, respectively); SY - sedentary young; SO — sedentary old; Ex-O — exercised old.

\section{Effect of aging and exercise on Ca-handling in ventricular myocytes \\ $\mathrm{Ca}^{2+}$ transients elicited in response to $\mathrm{Ca}^{2+}$} currents at different potentials were recorded in order to assess the efficiency of LTCC-RyR coupling in left ventricular myocytes. $\mathrm{I}_{\mathrm{CaL}}$ parameters were not significantly different between SY, SO and Ex-O ventricular myocytes (Fig. 4B). However, a significant increase in $\mathrm{Ca}^{2+}$-transients was characterized with advanced age both in exercised and non-exercised old groups' myocytes which implicate a higher gain of calcium-induced calcium release in both SO and Ex-O groups with respect to sedentary young myocytes, (Fig. 4A).

$\mathrm{SR} \mathrm{Ca}^{2+}$ content was estimated by measurement of caffeine-induced $\mathrm{Ca}^{2+}$ transient amplitude and calculation of the integral of the caffeineinduced NCX current $\left(\mathrm{I}_{\mathrm{NCX}}\right)$ obtained during $10 \mathrm{mM}$ caffeine application for $10 \mathrm{~s}$ (Fig. 5). Aging led to considerable increase in caffeine-induced $\mathrm{Ca}^{2+}$-transient amplitude and integrated current which infers a greater $\mathrm{Ca}^{2+}$ content in SR stores of $\mathrm{SO}$ group cardiac myocytes. Swimming exercise did not change further the $\mathrm{I}_{\mathrm{NCX}}$ current and SR content of ventricular myocytes in old rats.

\section{NOX-4 content, aconitase activity, and GSH/GSSG levels}

To examine whether oxidative stress parameters change with aging or swimming exercise NOX-4 concentration and aconitase activity were quantified by enzyme immunoassay kit (Fig. 6A-C). Compared to hearts from young female rats, NOX-4 was significantly increased in aged hearts. Exercise training did not change the elevated level of NOX-4 in aged hearts. However, aconitase activity was found to be significantly declined in elder groups and swimming exercise did not induce any remarkable alteration in cardiac aconitase activity of the aged female rats.

On the other hand, GSH/GSSG ratio was upregulated in hearts from Ex-O rats with respect to SO group. However, there was only a modest and 


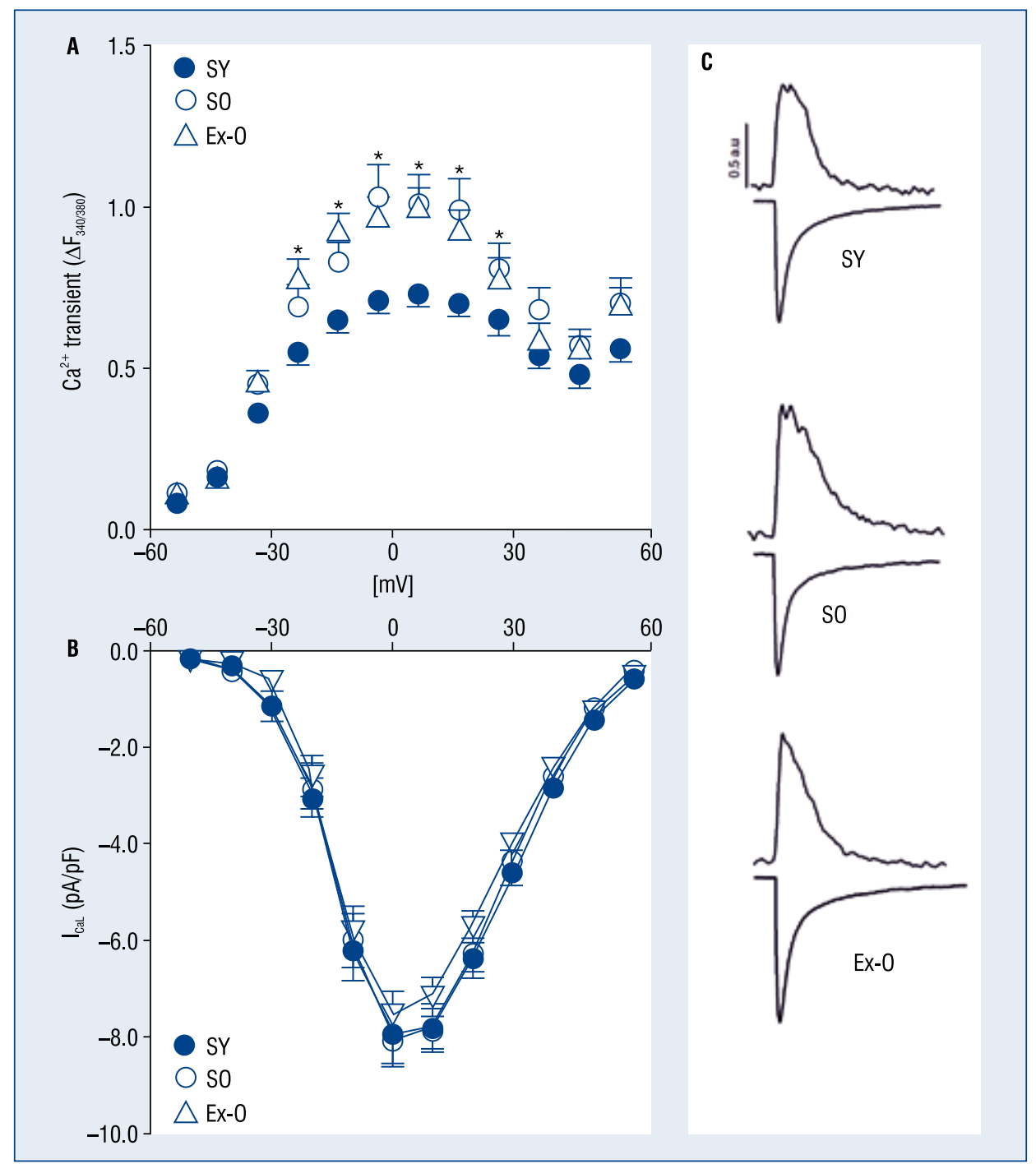

Figure 4. L-type $\mathrm{Ca}^{2+}$ current $\left(\mathrm{I}_{\mathrm{CaL}}\right)$ and associated $\mathrm{Ca}^{2+}$ transients obtained from ventricular myocytes in response to step pulses between $-50 \mathrm{mV}$ and $+60 \mathrm{mV}$; A. Average amplitudes of intracellular $\mathrm{Ca}^{2+}$ transients; $\mathbf{B}$. Peak $\mathrm{I}_{\mathrm{CaL}}$ densities as a function of membrane potential; $\mathbf{C}$. Representative traces of current and associated $\mathrm{Ca}^{2+}$ transients induced by a depolarizing step from to $0 \mathrm{mV}$ in sedentary young (SY), sedentary old (SO), and exercised old (Ex-O) group ventricular myocytes. Data are presented as mean $\pm \mathrm{SEM}$; ${ }^{*} \mathrm{p}<0.05 \mathrm{vs}$. SY group (number of cells, $n=33$ [9 hearts], 27 [11 hearts], 29 [6 hearts] for SY, SO and Ex-O groups, respectively).

non-significant reduction in GSH/GSSG values of SO group with respect to SY group.

\section{Discussion}

There are several major findings of this study. First, the contractile function of aged myocytes decreased significantly despite elevated $\mathrm{Ca}^{2+}$ transients and SR-Ca ${ }^{2+}$ load. Second, although swimming exercise did not change further the increased values of $\mathrm{Ca}^{2+}$ parameters in myocytes of aged rats, it improved cardiac contractile function remarkably. Third, moderate swimming exercise improved the impaired myofibril fine structure of senescent rats' myocardium. Fourth, exercise training did not change the elevated NOX-4 content and the depressed aconitase activity of aged hearts, while it elicited significant increase in GSH/GSSG ratio. Therefore, the present study demonstrated that swimming exercise does not exhibit significant effects on age-related alterations of intracellular $\mathrm{Ca}^{2+}$ handling in left ventricular myocytes of female rats, although it improves structural and contractile abnormalities of aged myocardium.

A growing body of evidence suggests that modification of the cardiac contraction with advanced age is 


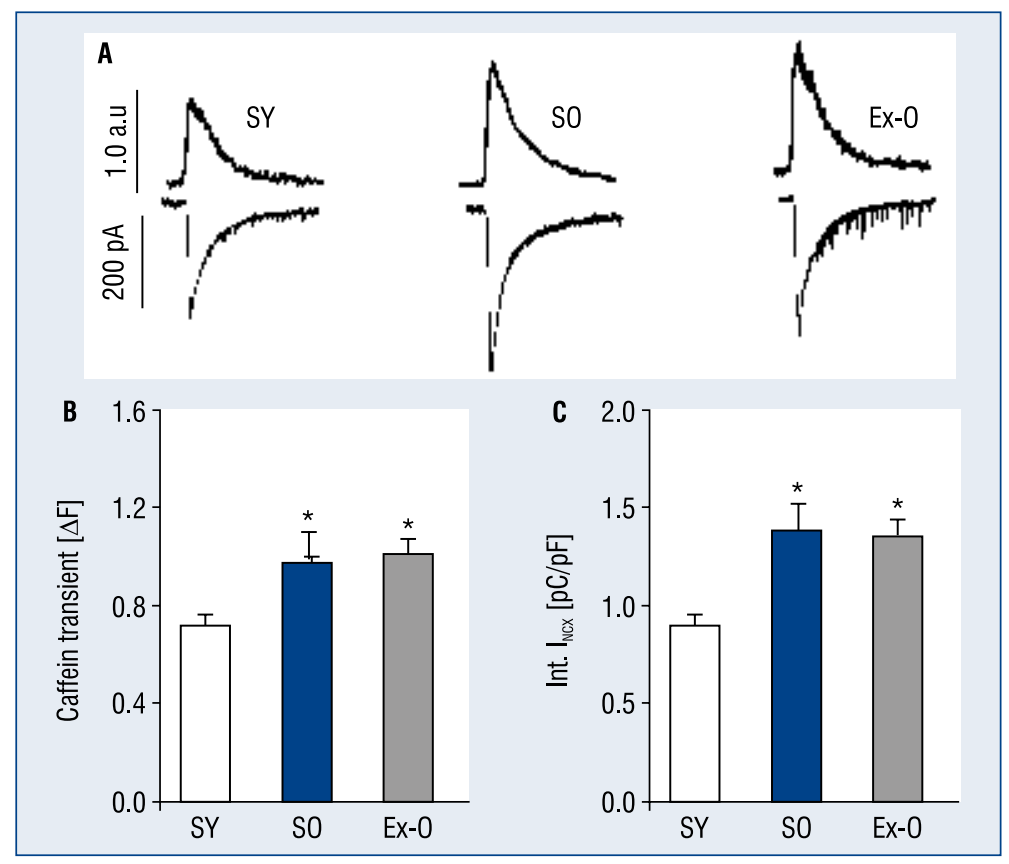

Figure 5. Caffeine-induced $\mathrm{Ca}^{2+}$ release and estimation of $\mathrm{SR} \mathrm{Ca}^{2+}$ content; $\mathrm{A}$. Representative raw tracings of $\mathrm{Ca}{ }^{2+}$ fluorescent signals and $\mathrm{Na}^{+}-\mathrm{Ca}^{2+}$ exchanger currents $\left(\mathrm{I}_{\mathrm{NCX}}\right)$ in myocytes after application of $10 \mathrm{mM}$ caffeine; B. Caffeineinduced $\mathrm{Ca}^{2+}$ transient amplitudes; C. Integral of the associated NCX current $\left(\int I_{\mathrm{NCX}}\right)$ after caffeine application. Data are presented as mean $\pm \mathrm{SEM}$; ${ }^{*} \mathrm{p}<0.05$ vs. SY group (number of cells, $n=23$ [8 hearts], 18 [9 hearts], 18 [6 hearts] for $\mathrm{SY}, \mathrm{SO}$ and Ex-O groups, respectively).

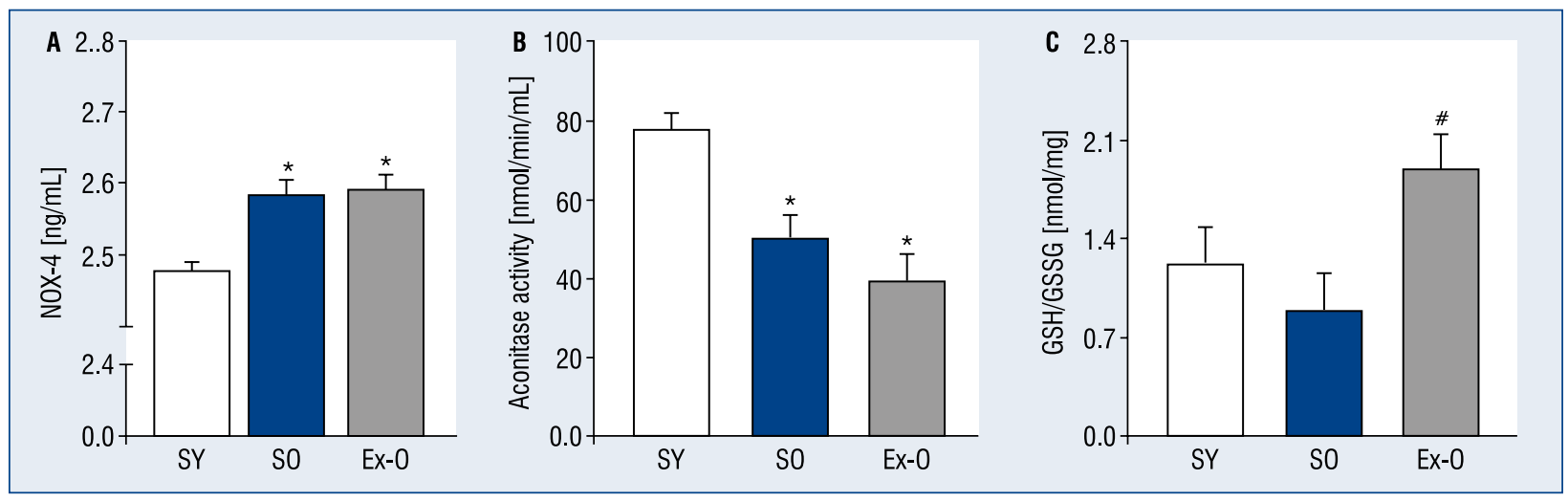

Figure 6. Myocardium NOX-4 content, aconitase activity, and tissue glutathione (GSH) and oxidized glutathione (GSSG) ratio presented as mean \pm SEM; * $p<0.05$ vs. sedentary young (SY) group; \#p $<0.05$ vs. sedentary old (SO) group ( $\mathrm{n}=7 \mathrm{SY}, 6 \mathrm{SO}, 7$ exercised old hearts).

associated with structural, functional and biochemical changes in the myocardium $[1,19]$. Exercise training is suggested in order to protect against age-induced remodeling of the left ventricle that includes myocyte loss, reactive hypertrophy of the remaining cells and increased myocardial fibrosis [20]. Consistent with these findings, we observed a reduction in agingdependent myofibril loss and impaired fine structure of myocardium in exercised rats.
$\mathrm{Ca}^{2+}$ handling alterations due to changes in the expression and/or function of $\mathrm{Ca}^{2+}$ regulatory proteins may also contribute to functional abnormalities observed in myocytes from aged hearts $[1,6,16]$. Accordingly, training dependent enhancement in systolic function of the aging heart has been ascribed to the improvement in $\mathrm{Ca}^{2+}$ handling of ventricular myocytes $[15,19]$. Despite discrepant findings reported for $\mathrm{I}_{\mathrm{CaL}}$ as 
a function of sex or animal species $[2,4,8,9,10]$ in the present study, neither age nor exercise was shown to alter the peak $\mathrm{I}_{\mathrm{CaL}}$ of left ventricular myocytes. However, $\mathrm{I}_{\mathrm{CaL}}$-triggered $\mathrm{Ca}^{2+}$ transients were higher in aged myocytes and swimming exercise did not change them further. Consistent with this, we observed significantly higher $\mathrm{SR} \mathrm{Ca}^{2+}$ content in cardiomyocytes as a function of age and exercise. Fares and Howlett [1] and Howlett [16] have also demonstrated an increase in SR $\mathrm{Ca}^{2+}$ content with aging in female cardiomyocytes, conversely to male rat myocytes. They [1] further demonstrated that contractile function does not deteriorate with age in myocytes both from female rats and mice, and thus suggested a likely mechanism by which cardiac contractile function is preserved in senescent females. Although we have observed similar increase in $\mathrm{Ca}^{2+}$ parameters of aged myocytes, the contractile function was reduced significantly. These results implicate that this increase in $\mathrm{SR} \mathrm{Ca}^{2+}$ content of aged myocytes is a compensatory response against the risk of contractile deficit, although it cannot counteract the effect of aging on systolic function. These data led us to suggest that intracellular $\mathrm{Ca}^{2+}$ changes cannot account for either diminishing effect of aging or improving effect of exercise on contractile function. Alternatively, increased myofilament $\mathrm{Ca}^{2+}$-sensitivity due to exercise may be the underlying mechanism of the improved contractility [21].

Reactive oxygen species, accumulation of which has been substantially accentuated with advanced age, can also lead to functional abnormalities [6]. Due to its high metabolic demand, heart is rich in mitochondria, and therefore susceptible to oxidative damage [22]. Apart from the ETC enzymes, NOXs may also actively contribute to ROS production in mitochondria. Of the NOXs currently known, NOX-4 has been suggested to drive oxidative stress in the mitochondria either alone or by stimulating ROS-induced ROS release, thereby exacerbating mitochondrial dysfunction which may result in cardiac dysfunction [13]. Recently, one study showing augmented NOX-4 expression in aged myocytes has gone further to propose a causal link between NOX-derived oxidative stress and decelerated contraction kinetics of cardiac myocytes from aged mice [6]. Consistently, in our study, NOX-4 was significantly increased in aged hearts but exercise training did not change the elevated level of NOX-4.

Furthermore, overexpression of cardiac NOX-4 in mice has been shown to inactivate aconitase [12] which is a mitochondrial enzyme specifically subjected to oxidative attack during aging [23]. Oxidative inactivation of aconitase can initiate a cascade resulting in dramatic increase in the oxidative damage to macromolecules including proteins [24]. With our study, we further support the idea that age-related accumulation of ROS may causally contribute to inactivation of aconitase and swimming exercise is unable to reverse this effect. However, the upregulation of GSH in exercised rats may protect the macromolecules against oxidative damage and reverses the structural abnormalities of myocardium in aged heart. As a consequence, swimming exercise stimulates the upregulation of antioxidant enzyme activity which counteracts the detrimental effects of ROS in myocardium and thereby improves the contractile function [6].

\section{Conclusions}

In summary, swimming exercise reverses the diminished myocyte contractile function despite the fact that it does not change further the altered $\mathrm{Ca}^{2+}$ homeostasis in aged cardiomyocytes. The cause of this is that contractile dysfunction of aged myocardium might result from structural abnormalities which are associated with elevated ROS and outweigh compensatory effect of upregulated $\mathrm{Ca}^{2+}$ parameters. Thus, it is likely that improved antioxidant capacity due to exercise training restores structural changes of myocardium and preserves contractile function of aged myocytes. On the other hand, increased myofilament $\mathrm{Ca}^{2+}$-sensitivity of exercised rats may also play role in restored contractile activity of aged myocardium. Further studies are required to fully characterize the exact mechanisms of exercise-induced improvements in aged myocardium.

\section{Acknowledgements}

This work was supported by The Scientific Research Projects Coordination Unit of Akdeniz University (project no. 2012.01.0103.009).

\section{Conflict of interest: None declared}

\section{References}

1. Fares E, Howlett SE. The impact of age on cardiac excitationcontraction coupling. Proceedings of the Australian Physiological Society, 2009; 40: 1-9.

2. Xiao RP, Spurgeon HA, O'Connor F, Lakatta EG. Age-associated changes in beta-adrenergic modulation on rat cardiac excitationcontraction coupling. J Clin Invest, 1994; 94: 2051-2059.

3. Ren J, Li Q, Wu S, Li SY, Babcock SA. Cardiac overexpression of antioxidant catalase attenuates aging-induced cardiomyocyte relaxation dysfunction. Mech Ageing Dev, 2007; 128: 276-285.

4. Isenberg G, Borschke B, Rueckschloss U. Ca2+ transients of cardiomyocytes from senescent mice peak late and decay slowly. Cell Calcium, 2003; 34: 271-280. 
5. Lim CC, Apstein CS, Colucci WS, Liao R. Impaired cell shortening and relengthening with increased pacing frequency are intrinsic to the senescent mouse cardiomyocyte. J Mol Cell Cardiol, 2000; 32: 2075-2082.

6. Rueckschloss U, Villmow M, Klockner U. NADPH oxidase-derived superoxide impairs calcium transients and contraction in aged murine ventricular myocytes. Exp Gerontol, 2010; 45: 788-796.

7. Grandy SA, Howlett SE. Cardiac excitation-contraction coupling is altered in myocytes from aged male mice but not in cells from aged female mice. Am J Physiol Heart Circ Physiol, 2006; 291: 26.

8. Liu SJ, Wyeth RP, Melchert RB, Kennedy RH. Aging-associated changes in whole cell $\mathrm{K}(+)$ and L-type $\mathrm{Ca}(2+)$ currents in rat ventricular myocytes. Am J Physiol Heart Circ Physiol, 2000; 279: H889-H900.

9. Walker KE, Lakatta EG, Houser SR. Age associated changes in membrane currents in rat ventricular myocytes. Cardiovasc Res, 1993; 27: 1968-1977.

10. Dibb KM, Rueckschloss U, Eisner DA, Isenberg G, Trafford AW. Mechanisms underlying enhanced cardiac excitation contraction coupling observed in the senescent sheep myocardium. J Mol Cell Cardiol, 2004; 37: 1171-1181.

11. Yang X, Sreejayan N, Ren J. Views from within and beyond: narratives of cardiac contractile dysfunction under senescence. Endocrine, 2005; 26: 127-137.

12. Ago T, Kuroda J, Pain J, Fu C, Li H, Sadoshima J. Upregulation of Nox4 by hypertrophic stimuli promotes apoptosis and mitochondrial dysfunction in cardiac myocytes. Circ Res, 2010; 106: 1253-1264.

13. Ago T, Matsushima S, Kuroda J, Zablocki D, Kitazono T, Sadoshima J. The NADPH oxidase Nox4 and aging in the heart. Aging, 2010; 2: 1012-1016.

14. Gwathmey JK, Slawsky MT, Perreault CL, Briggs GM, Morgan JP, Wei JY. Effect of exercise conditioning on excitation-contraction coupling in aged rats. J Appl Physiol, 1985; 69: 1366-1371.
15. Tate CA, Taffet GE, Hudson EK, Blaylock SL, McBride RP, Michael LH. Enhanced calcium uptake of cardiac sarcoplasmic reticulum in exercise-trained old rats. Am J Physiol, 1990; 258: H431-H435.

16. Howlett SE. Age-associated changes in excitation-contraction coupling are more prominent in ventricular myocytes from male rats than in myocytes from female rats. Am J Physiol Heart Circ Physiol, 2010; 298: 4.

17. Ozdemir S, Bito V, Holemans P, Vinet L, Mercadier JJ, Varro A, Sipido KR. Pharmacological inhibition of na/ca exchange results in increased cellular $\mathrm{Ca} 2+$ load attributable to the predominance of forward mode block. Circ Res, 2008; 102: 1398-1405.

18. Ozturk N, Yaras N, Ozmen A, Ozdemir S. Long-term administration of rosuvastatin prevents contractile and electrical remodelling of diabetic rat heart. J Bioenerg Biomembr, 2013; 45: 343-352.

19. Spurgeon HA, Steinbach MF, Lakatta EG. Chronic exercise prevents characteristic age-related changes in rat cardiac contraction. Am J Physiol, 1983; 244: H513-H518.

20. Kwak HB, Song W, Lawler JM. Exercise training attenuates ageinduced elevation in $\mathrm{Bax} / \mathrm{Bcl}-2$ ratio, apoptosis, and remodeling in the rat heart. Faseb J, 2006; 20: 791-793.

21. Wisløff U, Loennechen JP, Falck G, Beisvag V, Currie S, Smith G, Ellingsen $\mathrm{O}$. Increased contractility and calcium sensitivity in cardiac myocytes isolated from endurance trained rats. Cardiovasc Res, 2001; 50: 495-508.

22. Dai DF, Rabinovitch PS, Ungvari Z. Mitochondria and cardiovascular aging. Circ Res, 2012; 110: 1109-1124.

23. Sohal RS. Mitochondria generate superoxide anion radicals and hydrogen peroxide. Faseb J, 1997; 11: 1269-1270.

24. Yan LJ, Levine RL, and Sohal RS. Oxidative damage during aging targets mitochondrial aconitase. Proc Natl Acad Sci USA, 1997; 94: 11168-11172. 